\title{
Case Report and Brief Review: Pterois volitans (Lionfish) Envenomation of the Hand: Taming A Lionfish Sting \\ A Lucerna $^{1}, \mathrm{~J} \mathrm{Espinosa}^{1}$, AB Norinsky ${ }^{2}$
}

\section{INTRODUCTION}

The lionfish is a member of the family Scorpaendia which comprises most of the world's venomous fish. Lionfish envenomations can be seen in divers, fisherman and swimmers. They are naturally found in tropical waters in the Indo-Pacific region. They are also often found in aquariums worldwide. We present the case of a 20 year male who presented to the emergency department (ED) after he was stung on his hand by a lionfish (Pterois volitans) while cleaning an aquarium.

Keywords: Lionfish envenomation; Emergency Department and lionfish envenomation; Pterois volitans envenomation; venomous fish; management of lionfish envenomation

From: ${ }^{1}$ Department of Emergency Medicine, Rowan University SOM Kennedy University Hospital, Stratford, NJ, USA, ${ }^{2}$ Department of Emergency Medicine, Houston Methodist Hospital-San Jacinto, Houston, Texas, USA.

Correspondence: Dr A Lucerna, Department of Emergency Medicine, SOM Kennedy University Hospital, Rowan University, 18 East Laurel Road, Stratford, NJ 08084, USA.

E-mail: primarysurvey@gmail.com 


\section{CASE REPORT}

A 20 year male presented to the emergency department ( ED ) two hours after he was stung by a lionfish ( Pterois volitans ) while cleaning an aquarium at his place of employment. The patient reported he was stung on the middle phalanx of the second digit, left hand, palmar aspect. The patient developed pain and swelling shortly after the envenomation. The pain had progressed despite taking $200 \mathrm{mg}$ of ibuprofen prior to arrival.

In the $\mathrm{ED}$, the patient was noted to have redness and swelling of the entire second digit of the left hand (Figures 1,2). The range of motion of all joints of the affected finger was within normal limits. The left hand was noted to have a normal finger flexion cascade and was neurovascularly intact. There were no visible spines or foreign bodies on examination of the affected area.

Initial vital signs were as follows: blood pressure 116/66 torr, heart rate 110 beats per minute, respiratory rate 22 breaths per minute, temperature 98.9 degrees F. and oxygen saturation $100 \%$ on room air. The patient denied any pertinent medical and surgical history. He also denied smoking, alcohol, or drugs.

The patient was given morphine sulfate intravenously for analgesia. The patient's tetanus vaccine was updated. A complete blood count, basic metabolic panel and C- reactive protein were unremarkable. The left hand x-ray did not show any bony abnormalities and no foreign bodies were noted.

A phone consultation was obtained with Poison Control. Immersion of the affected hand in water-- no warmer than 114 degrees Fahrenheit---for 30-90 minutes was recommended. An orthopedic consultation recommended the same initial treatment. Empiric antibiotics were 
provided under the guidance of the Infectious Disease service to cover Vibrio and Aeromonas species as well skin flora: vancomycin, ceftriaxone and ciprofloxacin were given intravenously.

The patient was admitted to the hospitalist service for pain management, monitoring for possible systemic manifestation of the envenomation and for neuro-vascular checks of the affected finger. A diagnosis of cellulitis was made, which improved with intravenous antibiotics. Tenosynovitis was ruled out by the Orthopedic Service. The patient was discharged within 48 hours of admission on oral amoxicillin-clavulanate and instructions to follow-up with primary care.

\section{DISCUSSION}

The lionfish is a member of the family Scorpaendia. The Scorpaendia family comprises most of the world's venomous fish (1). Within this family is the lionfish--Pterois genus, principally Pterois volitans. It is noteworthy that another member of the Scorpaendia family, the stonefish, is considered the most venomous fish in the world, with a toxin that resembles cobra venon in toxicity (2). Midway in toxicity between the lionfish and the stonefish is the scorpionfish. The least toxic member of this toxic trio, the lionfish, is capable of causing significant local and systemic manifestations.

Lionfish are quite beautiful in appearance and are often found in aquarium settings worldwide. As well-stated by Patel, "Masterfully camouflaged in its rocky environment, the lionfish is as beautiful as it is voracious and poisonous" (3). 
This beauty has made the lionfish one of the top 10 aquarium fish. (4) Lionfish envenomations can occur in association with aquarium cleaning and fish handling in an aquarium setting and thus often occur on the hands (5).

However, world-wide, lionfish envenomations can be seen in divers, fisherman and swimmers (6). They are naturally found in tropical waters in the Indo-Pacific region, although there has been remarkable and progressive proliferation into more temperate waters. Lionfish are increasing found in the coastal waters of the Caribbean and the eastern shores of South America - and more recently have been found in the waters off the coast of Florida, Georgia, North Carolina, New Jersey and New York. $(1,6)$ As an odd fact, Hurricane Andrew damaged an aquarium in the Miami area in 1992, releasing six adult lionfish into the waters of Biscayne Bay. In the following months, lionfish were reported off the coast of Florida (6).

Resiere notes that in the native environment of the the tropical waters of the Indo-Pacific region, the lionfish is contained in proliferation by natural predators. However, lionfish are known to proliferate in non-native areas, especially since the year 2000. "This spectacular invasion may have dramatic ecological repercussions," according Resiere et al, since lionfish can decimate populations of local native small fish. His study occurred in the French West Indies where the lionfish has been proliferating. The fish is not generally an inhabitant of shallow waters, and hence divers and fisherman are affected more commonly than swimmers $(1,6)$. There is said to be no risk to bathers in shallow waters (1).

The venom of the lionfish is a heat-labile protein. Lionfish spines, with the exception of the pectoral spines, are capable of envenomation (5). The majority of envenomations associated with aquarium contact are to the hand, as was noted in the case presented. 
The pain is generally immediate and reaches a peak within two hours. The pain can be severe and typically persists for 6 to 12 hours---hence, as noted by Garyfallos, the maritime folklore saying that the pain of a lionfish envenomation lasts until the turning of the tide.

Inflammation is a hallmark, leading to edema and warmth. Pain can involved the entire affected extremity (3). Elevation of the affected extremity may help reduce edema, if present (4). Vesicles and blisters may occur with rare case reports of skin necrosis. Systemic manifestations have been described, including a wide variety of symptoms including nausea and vomiting, diaphoresis, abdominal pain, hypotension, and syncope. Significant systemic symptoms are noted to be rare, overall (5).

A grading system of severity of envenomation in reference to the affected skin has been described. (Grade 1: erythema and edema, Grade 2: vesicles and blisters Grade 3: skin necrosis.) In the large series by Resiere et al, Grade 1 injuries were most common (71\%), Grade 2 effects were seen in $26 \%$ of cases and Grade 3 in $3 \%$ of cases (6).

The nature of the venom has been the subject of research. Nitric oxide release in relation to envenomation has been described. Adrenergic and cholinergic activity appears to contribute to systemic symptoms in a variety of animal models in an elegant study of lionfish venom (7).

It is possible to sustain a puncture from a venom-bearing fin and not any experience any local or systemic symptoms. This is has been described by Satora as an "empty sting" and may be due to an abnormality of the venom organ associated with the puncturing fin. Empty stings were seen in three of fourteen tank-bred lionfish stings in a series presented from a Poison Control Center in Poland (8). 
The lionfish can envenomate up to 48 hours after it dies-thus envenomation can occur when an aquarium owner at home or a worker in a tropical fish store attempts to remove a dead lionfish from a fishtank (3).

The literature seems to be consistent in the recommendation that lionfish envenomation is treated with immersion of the affected extremity, typically the hand, into warm water. The water is recommended to be nonscalding - up to 45 degrees Celcius - for 30 to 90 minutes or until the pain resolves (3). This was the treatment provided in our case.

The reason for the general improvement in symptoms with hot water immersion may not be as simple as denaturation of the venom protein - since pain may return after the extremity is removed from the hot water after 30 to 90 minutes (4). The heat itself may over-ride the vasoconstrictive effect of the venom (1). There is some evidence that the vesicles and blister fluid seen in Grade 2 envenomations may contain residual venom - and thus evacuation of vesicles and blisters should be considered (9).

An important consideration is that the water temperature should be closely monitored. This is of especial importance as a safety issue if the affected extremity has been anesthetized (local anesthesia) for pain relief and/or to facilitate the examination and removal of foreign bodies. In a series of 117 lionfish envenomations, analgesics were, for the most part, given systemically (6).

Local anesthesia facilitates inspection, irrigation and removal of any residual foreign bodies from a wound. One author described the use of a long-acting anesthetic given as a digital block (bupivacaine) in a lionfish envenomation to the hand (4). Retained lionfish spines are not only a foreign body - they may also contain residual venom. Imaging may help to identify 
retained spine material. The literature most often recommends plain films, however other imaging modalities, such as ultrasound, CT and MRI modalities have been used.

Given the nature of a lionfish wound, tetanus prophylaxis should be provided if indicated (2).

The Resiere series of lionfish envonomations suggests an overall good prognosis---but cautions attention to potential morbitities. One hundred percent of cases in the series of 117 envenomations had pain and edema, as was seen in our case. Local infection was seen in $18 \%$ of cases, including cellulitis, skin necrosis, skin abscess and septic arthritis. Cellulitis was identified in the case presented. The series makes it clear that the range of systemic manifestations of lionfish venom is remarkably wide and includes parasthesias (90\%), abdominal cramping (62\%), tachycardia (34\%), hypertension (21\%), hypotension (18\%) and bradycardia in $3 \%$. Hypophosphatemia can occur. Transaminases can be elevated. Transient weakness occurred in $27 \%$ of cases (6).

Overall, the treatment of lionfish envenomation is relatively pragmatic. Resiere calls for randomized clinical trials "to improve the level of evidence of the different techniques traditionally used to manage marine envenomation."

In reference to prevention of lionfish injurires, it is noteworthy that latex gloves do not prevent envenomation (5). The venom protein is antigenic (5). Several authors, including Patel et al, caution that repeat envenomation by a lionfish may cause an anaphylactic reaction-and caution against all future direct lionfish contact $(3,5)$. 


\section{CONCLUSIONS}

We present the case of a 20 year male who presented to the emergency department (ED) two hours after he was stung on his hand by a lionfish (Pterois volitans) while cleaning an aquarium at his place of employment. The patient developed pain and swelling shortly after the envenomation. The patient was managed in consulation with the Poison Control and Orthopedics services, who recommended immersion of the affected hand in water-- no warmer than 114 degrees Fahrenheit---for 30-90 minutes was recommended, morphine sulfate intravenously for analgesia, empiric antibiotic coverage and updating the tetanus vaccine. The left hand $\mathrm{x}$-ray did not show any bony abnormalities and no foreign bodies were noted. Empiric antibiotics were given. The patient was admitted to the hospitalist service for pain management, monitoring for possible systemic manifestation of the envenomation and for neuro-vascular checks of the affected finger. A diagnosis of cellulitis was made, which improved with intravenous antibiotics. Tenosynovitis was ruled out by the Orthopedic Service. The patient was discharged within 48 of admission with an out-patient (amoxicillin-clavulanate) prescription and follow-up with primary care. The case report reviews lionfish envenomation management.

The lionfish is a member of the family Scorpaendia. The least toxic member of this toxic trio, the lionfish, is capable of causing significant local and systemic manifestations. Lionfish one of the top 10 aquarium fish. Lionfish envenomations can occur in association with aquarium cleaning and fish handling in an aquarium setting and thus often occur on the hands. However, world-wide, lionfish envenonmations can be seen in divers, fisherman and swimmers.

The case report reviews current thinking concerning lionfish envenomation presentation and management. 


\section{REFERENCES}

1. Haddad V Jr, Stolf HO, Risk JY, França FO, Cardoso JL. Report of 15 injuries caused by lionfish (pterois volitans) in aquarists in Brazil: a critical assessment of the severity of envenomations. J Venom Anim Toxins Incl Trop Dis 2015; 21: 8. doi: 10.1186/s40409015-0007-x. eCollection 2015.

2. Kasdan ML, Kasdan AS, Hamilton DL.Lionfish envenomation. Plast Reconstr Surg 1987; 80: 613-4.

3. Patel MR, Wells S.Lionfish envenomation of the hand. J Hand Surg Am 1993; 18: 5235.

4. Garyfallos GT, Madden JF. Lionfish envenomation. Ann Emerg Med 1996; 28: 456-7.

5. Vetrano SJ, Lebowitz JB, Marcus S.Lionfish envenomation. J Emerg Med 2002; 23: $379-82$.

6. Resiere D, Cerland L, De Haro L, Valentino R, Criquet-Hayot A, Chabartier C, Kaidomar S, Brouste Y Mégarbane B, Mehdaoui H.Envenomation by the invasive Pterois species volitans (lionfish) in the French West Indies--a two-year prospective study in Martinique. Clin Toxicol (Phila) 2016; 54:313-8.

7. Church JE, Hodgson WC.Adrenergic and cholinergic activity contributes to the cardiovascular effects of lionfish (Pterois volitans) venom. Toxicon 2002; 40: 787-96.

8. Satora L. Lionfish envenomations in Poland. Przegl Lek 2009; 66: 285-6.

9. Auerback PS, McKinney HE, Rees RS et al: Analysis of vesicle fluid following the sting of the lionfish Pterois volitans. Toxicon 1987; 25: 350-51. 


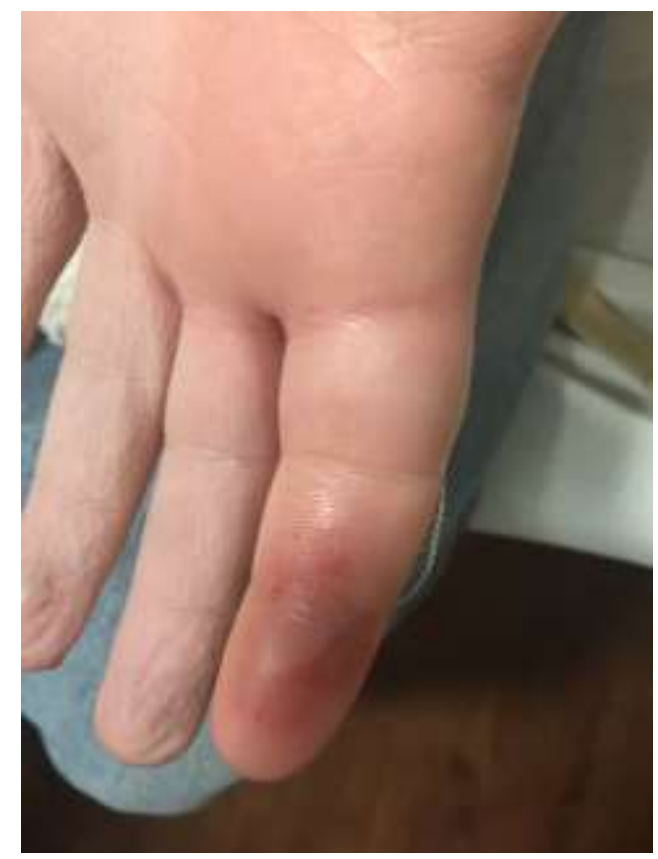

Fig. 1: Palmar aspect of the second digit of the left hand showing edema and erythema of the middle and distal phalanx.

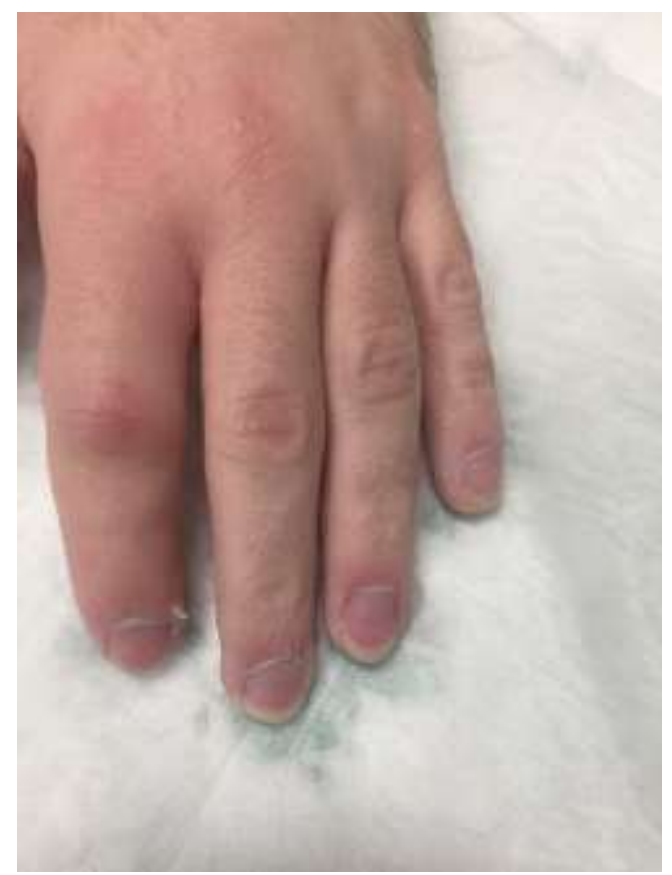

Fig. 2: Dorsal aspect left hand second digit showing edema and erythema tracking up to the metacarpophalangeal region. 